\title{
Editorial
}

\section{Proceedings of the Second International Conference of the French-speaking Society for Theoretical Biology}

\author{
Julien Arino - Stéphanie Portet
}

Received: 5 October 2009/Accepted: 12 October 2009/Published online: 24 October 2009

(C) Springer Science+Business Media B.V. 2009

The Second International Conference of the French-speaking Society for Theoretical Biology (SFBT) was held June 4-6 2007 in Winnipeg, Manitoba, Canada. It followed the May 2004 First International Conference of the SFBT, held in Marrakesh, Morocco, and will be followed by the Third International Conference of the SFBT, which will take place in Tunis in June 2010.

The SFBT has been meeting yearly since 1985, most frequently in Saint Flour, Cantal, France. These meeting are still held in years where the International Conference is not taking place. During the Annual Meetings and the International Conferences, the participation of students is emphasized by the attribution of a prize for best student poster and/or presentation. In Winnipeg, the René Thom prize was awarded for the first time (Thom was a founding member of SFBT). Recipients of the prize were Virginie LeRolle (best presentation) and Peng T. (Troy) Teo (best poster).

The range of topics discussed during SFBT events is quite broad, covering many aspects of theoretical biology ranging from complex mathematical and physical problems to philosophical considerations. The articles published in this special issue serve to illustrate this diversity.

Jacques Demongeot considers the notion of biological age, which he opposes to the notion of chronological age. He argues that the number of divisions that a cell has undergone and can still undergo is better suited for the description of cell age, but also of organ age, and presents applications of this notion in various contexts. Alexander Dibrov et al's contribution deals with a problem of computational chemistry in the Life Sciences. They propose a new method to rank protein-protein interaction models using free energy minimization and present a rational method for

J. Arino $(\bowtie) \cdot S$. Portet

Department of Mathematics, University of Manitoba, Winnipeg, MB, Canada

e-mail: arinoj@cc.umanitoba.ca

S. Portet

e-mail: portets@cc.umanitoba.ca 
choosing convergence criteria when comparing two structures. Etienne Farcot and Jean-Luc Gouzé provide further results about a system they previously considered that describes gene regulatory networks. The problem that they consider is difficult because of the piecewise continuous nature of the vector field, and their results are quite profound. Virginie LeRolle et al. consider an electromechanical simulation model for the heart's left ventricle. This very detailed model mixes different formalisms and represents the system as consisting of twelve different subsystems, but it is striking that despite its complexity, real data was used to calibrate the model. Ángeles Rincón et al. consider stochastic metapopulation models operating on two time-scales, with the movement between physical locations being much faster than the demographic processes within the locations. They study the relation between the original model and the so-called reduced model in which the fast timescale has been eliminated. Finally, Mansour Serghini et al. formulate a sizestructured population dynamics model for the evolution of the distribution of sardines on the Moroccan coast, and use data to find the value of the growth rate of the population. They consider the sensitivity of this growth rate on parameters of the model and propose strategies to raise this growth rate, which they find to have a value less than one (implying ceteris paribus a reduction of the population).

Acknowledgments JA, SP and the SFBT wish to thank the Faculty of Science at the University of Manitoba, the University of Manitoba, MITACS, the Gouvernement du Québec and the Manitoba Government for their financial support of the event. Readers interested in learning more about SFBT and its activities can consult its web site at http://www.sfbt.org. 